\title{
Solar and magnetospheric forcing of the low latitude thermospheric mass density as observed by CHAMP
}

\author{
S. Müller ${ }^{1, *}$, H. Lühr ${ }^{2}$, and S. Rentz ${ }^{2}$ \\ ${ }^{1}$ Free University Berlin, Institute for Meteorology, Carl-Heinrich-Becker Weg 6-10, 12165 Berlin, Germany \\ ${ }^{2}$ Helmholtz-Centre Potsdam, GFZ - German Research Centre for Geosciences, Telegrafenberg, 14473 Potsdam, Germany \\ *now at: Norwegian Meteorological Institute, P.O. Box 6314, 9293 Troms $\varnothing$, Norway
}

Received: 19 November 2008 - Revised: 9 April 2009 - Accepted: 15 April 2009 - Published: 6 May 2009

\begin{abstract}
We have studied the dependence of the thermospheric mass density at equatorial latitudes on the influence of various drivers. This statistical study is based on CHAMP accelerometer measurements. Our aim is to delineate the influences of the different contributions. For the isolation of the effects we make use of a dedicated data selection procedure and/or removal of disturbing effects. In a first step all readings are normalised to an altitude of $400 \mathrm{~km}$. For the investigation of the solar influences only magnetically quiet days $\left(A_{p} \leq 15\right)$ are considered. The dependence on solar flux can well be described by a linear relation within the flux range F10.7=80-240. The slope is twice as steep on the day side as on the night side. The air density exhibits clear annual and semi-annual variations with maxima at the equinoxes and a pronounced minimum around June solstice. The thermosphere maintains during quiet days a day to night mass density ratio very close to 2 , which is independent of solar flux level or season. The magnetospheric input causing thermospheric density enhancement can well be parameterised by the $a_{m}$ activity index. The low latitude density responds with a delay to changes of the index by about $3 \mathrm{~h}$ on the dayside and $4-5 \mathrm{~h}$ on the night side. The magnetospheric forcing causes an additive contribution to the quiet-time density, which is linearly correlated with the $a_{m}$ index. The slopes of density increases are the same on the day and night sides. We present quantitative expressions for all the dependences. Our results suggest that all the studied forcing terms can be treated as linear combinations of the respective contribution.
\end{abstract}

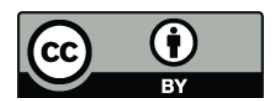

Correspondence to: S. Müller (sevim.mueller@met.no)
Keywords. Atmospheric composition and structure (Pressure, density, and temperature) - Ionosphere (Ionosphereatmosphere interactions) - Meteorology and atmospheric dynamics (Thermospheric dynamics)

\section{Introduction}

The thermospheric density is known to be a highly variable quantity. It responds to variations of geophysical conditions in a rather complex way. This is in particular valid during times of enhanced magnetic activity (Prölss, 1997). Atmospheric models try to reproduce the thermospheric conditions as close as possible with the help of suitable parameters. Rather important are the dependences on local time and season. External drivers are the short wavelength solar radiation and the solar wind input to the magnetosphere with its subsequent release of energy to the thermosphere. These are reflected by the four parameters that are required when running atmospheric models. For example, in case of the MSIS models (Hedin, 1983) the input data needed are the coordinates of the measurement point, time (UT), solar flux level (F10.7) and magnetic activity $\left(a_{p}\right)$. The quality of a model depends on the ability to reflect the response of the thermosphere to all the listed parameters properly. Some of the parameters may even interact non-linearly. Furthermore, there may be additional controlling forces that are not considered in the parameter set.

In this study we are going to make use of four years of thermospheric mass density observations to investigate the response to the above mentioned parameters in a statistical approach. The mass density data have been derived from the accelerometer measurements on board the CHAMP satellite.

Published by Copernicus Publications on behalf of the European Geosciences Union. 
The procedures for estimating the air density have previously been introduced (e.g., Bruinsma et al., 2004; Liu et al., 2005).

The general features of the thermospheric density and their global distribution, as observed by CHAMP, have, for example, been described by Liu et al. (2005). Their study is limited to the observations of the year 2002. For the interpretation the data set was sorted according to the magnetic activity. They considered two groups, one in the $K_{p}$ range $0-2$ and the other $K_{p}=3-4$. From the observed differences a qualitative impression of the influence of magnetic activity on the thermosphere could be gained.

A rather comprehensive study about the effects of solar extreme ultra-violet (EUV) radiation on the thermospheric density was presented by Guo et al. (2007). These authors investigated the correlation of observed density variations with various proxies for the solar EUV radiation. They also tested combinations of these proxies. The analysis was performed separately for high, middle and low latitudes zones. Best correlations resulted from low latitude readings. Unfortunately, the authors did not distinguish in their analysis between local time sectors from which the density measurements were taken.

In recent years the seasonal variation of the thermospheric density has been addressed in several studies (e.g. Bowman, 2004; Guo et al., 2008). In a comprehensive study Bowman (2004) analysed the orbit evolution of 13 space objects and deduced from it the semi-annual variation of the mass density in the altitude range 220 to $1100 \mathrm{~km}$ over the years 1969 through 2002. When comparing the ratio of the derived major equinox maximum with the major minimum around June solstice he found an increase from $65 \%$ to $125 \%$ from solar minimum to maximum years for heights around $400 \mathrm{~km}$. No data selection by magnetic activity or solar flux level was applied to the data. Thus significant year-to-year variations of the semi-annual amplitude are observed.

Based on daily averages of thermospheric densities derived from CHAMP accelerometer measurements Guo et al. (2008) deduced the intra-annual variability for the years 2002 through 2005. In order to account for the altitude, local time, latitude, magnetic activity and solar flux dependence of the mass density they subtracted the local prediction of the JB2006 model (Bowman et al., 2008) from each CHAMP reading. For this exercise the seasonal dependence module in the model was deactivated. Unfortunately, the model did not capture all the natural variations properly. Therefore some empirical corrections were applied in order to reduce the remaining local time and solar flux dependences of the residual data. From a spectral analysis of the density residuals they obtain a significant year-to-year variability of the first four annual harmonics. According to their results most of the spectral changes can be explained by variations of the solar EUV flux. Opposed to that magnetic activity seemed to play only a minor role for the seasonal variation.

The response of thermospheric density to magnetic activity was studied recently by Lathuillère et al. (2008). They considered one year (2004) of CHAMP data. In order to isolate the response to magnetic activity they had to compensate for the other influences. The solar flux effect was removed by normalising the density data with respect to the MgII index rather than F10.7. The day-to-day variability was taken into account with the help of a singular value decomposition approach (Menvielle et al., 2007). Remaining variations showed a clear dependence on magnetic activity.

Although these various studies addressed different aspects of the thermospheric density response to influencing conditions there is no comprehensive study considering simultaneously all four mentioned parameters (local time, solar flux, season and magnetic activity). This is needed to find the functional dependence of the density on each of the parameters separately, and to investigate whether there are interlinks between the dependences.

Our approach for answering these open questions is to make use of four years of CHAMP accelerometer measurements. We think, this long and continuous time series enables us to delineate the influences of the four parameters considered.

\section{Data processing}

The CHAMP satellite, launched on 15 July 2000 , cycles the Earth on a near-polar (inclination $87.25^{\circ}$ ) and almost circular orbit at an altitude of about $400 \mathrm{~km}$ (Reigber et al., 2002). The orbit has decayed from $445 \mathrm{~km}$ at the beginning of 2002 to $365 \mathrm{~km}$ at the end of 2005 . The orbital plane precesses through one hour of local time in eleven days, thus it takes CHAMP 131 days to cover all local times.

We analyse the readings taken by the STAR (Space Threeaxes Accelerometer for Research missions) accelerometer (ACC) during the period 1 January 2002 to 31 December 2005. In total there are more than 45000 equator crossings available. The data considered are the pre-processed Level-2 ACC data. These are averages over $10 \mathrm{~s}$. Another preparation step is the removal of the acceleration due to solar radiation pressure.

The acceleration (deceleration), $\boldsymbol{a}$, due to air drag can be expressed as

$\boldsymbol{a}=\frac{1}{2} \rho \frac{C_{D}}{m} A_{\mathrm{eff}} V^{2} \widehat{\boldsymbol{v}}$

where $\rho$ is the local thermospheric mass density, $C_{D}$ the drag coefficient, $m$ the spacecraft mass, $V$ its velocity relative to the air at rest and $\widehat{v}$ is the unit vector of the velocity in ram direction. We calculate the effective area in ram direction as: $A_{\text {eff }}=A_{x} \cos \alpha+A_{y} \sin |\alpha|$, where $\alpha$ is the side slip angle in the horizontal plane, calculated from the ratio of the acceleration components, $\tan \alpha=\frac{a_{y}}{a_{x}}$.

Equation (1) can be solved for the mass density, $\rho$

$\rho=\frac{2 m \boldsymbol{a} \cdot \widehat{\boldsymbol{v}}}{C_{D} A_{e f f} V^{2}}$ 
where we have used for $m$ the actual mass of CHAMP $(\sim 500 \mathrm{~kg})$; for $V$ we consider the orbital velocity $(7.6 \mathrm{~km} / \mathrm{s})$ and in across-track direction the corrotation plus the zonal wind velocity. For the drag coefficient we used the ESArecommended value $C_{D}=2.2$. The resolution offered by the accelerometer is $3 \times 10^{-9} \mathrm{~m} / \mathrm{s}^{2}$. When inserting these values into Eq. (2) and considering a typical value for the area, $A_{\text {eff }}=0.8 \mathrm{~m}^{2}$, we obtain a resolution of the mass density of $6 \times 10^{-14} \mathrm{~kg} / \mathrm{m}^{3}$.

An important quantity influencing the thermospheric density is the EUV radiation. A commonly used index for quantifying the solar flux level is the F10.7 value. Past studies have, however, shown that the composite index P10.7 $=0.5$ $\left(\mathrm{F} 10.7+\mathrm{F} 10.7_{81 \text { days }}\right)$ is more appropriate for describing the thermospheric, ionospheric energy input (e.g., Guo et al., 2007; Liu et al., 2006). These authors report that the correlation with the air density is even improved if the P10.7 value from the previous day is used. Throughout this study the solar flux level is approximated in this way.

Figure 1a shows the occurrence number of orbits for high and low solar flux conditions during the relevant local time hours. The same distribution is valid for the night side. The number of samples is systematically higher for low solar flux $(\mathrm{P} 10.7<130$, blue). However, all half hourly bins are filled with more than 250 samples. In case of a separation by magnetic activity one can read from Fig. $1 \mathrm{~b}$ that about two-third of the samples fall into the quiet time bin $\left(a_{p}<15\right)$. These many samples from quiet days provide a good basis for studying the solar forcing of the thermosphere.

Based on this dedicated data set a statistical analysis of the thermospheric characteristics is performed.

\section{Statistical analysis}

Our approach for identifying the dependence of the thermospheric density on the various parameters is to make use of a large amount of observations and determine statistically significant properties. For this purpose we try to isolate the individual effects as good as possible and quantify the responses one after the other.

The considered time interval spans the years 2002 through 2005. Within that period CHAMP visited all local times more than 11 times. We limit our investigations here to the low latitude band of $\pm 30^{\circ}$ geographical latitude. For each of the 22762 orbits we calculate one low latitude average density value from the upleg and another from the downleg arc. The width of the latitude band has been chosen to be large enough to cover the seasonal variation of the sub-solar point and on the other hand to be sufficiently far away from regions of direct magnetospheric energy input at high latitudes. Furthermore, it is identical with the low latitude band chosen by Guo et al. (2007), thus making results directly comparable. It may be noted in this context that this latitude band covers

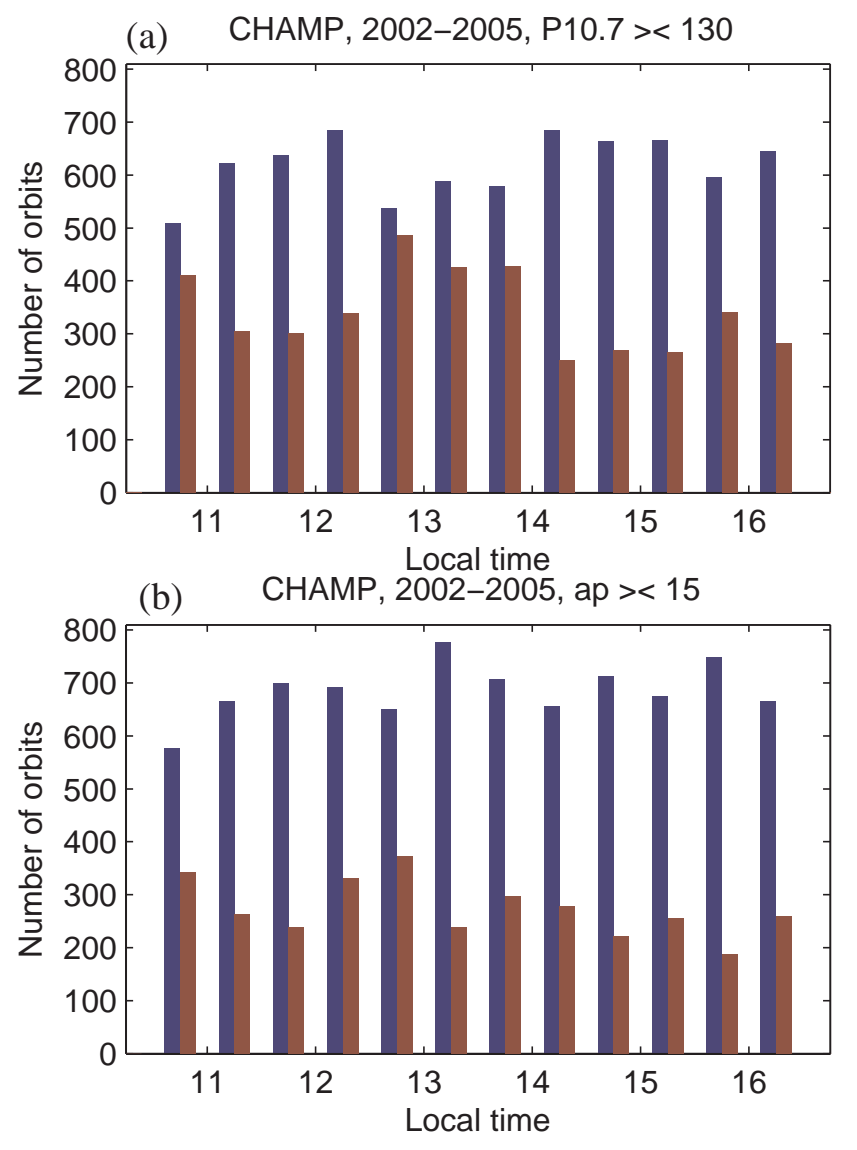

Fig. 1. Sample distribution of the considered density values over the relevant local times in half-hour bins. Counts are shown for high (red) and low (blue) (a) solar flux, P10.7, periods and (b) magnetic activity, $a_{p}$, levels.

$50 \%$ of the Earth's surface, thus represents a significant part of the thermosphere.

In the next processing step we have normalised the observed densities to a common altitude of $400 \mathrm{~km}$. This is necessary in order not to confuse height related changes with other dependencies. For this purpose we used the model NRLMSISE-00 (Picone et al., 2002). The employed procedure is

$\rho(400)=\rho(h) \frac{\operatorname{MSIS}(400)}{\operatorname{MSIS}(h)}$

where $\rho(h)$ is the recorded density, $\operatorname{MSIS}(400)$ and $\operatorname{MSIS}(h)$ are the predicted densities at $400 \mathrm{~km}$ and at measurement height, $h$, respectively. For this purpose the model has been run with the actual input parameters, $a_{p}$ and F10.7, valid for the considered epoch. Since the altitude of CHAMP did not vary much over the considered period $(445-365 \mathrm{~km})$ the normalisation to a common height is less than the scale height $(H \sim 60 \mathrm{~km})$. 
CHAMP, 2002-2005
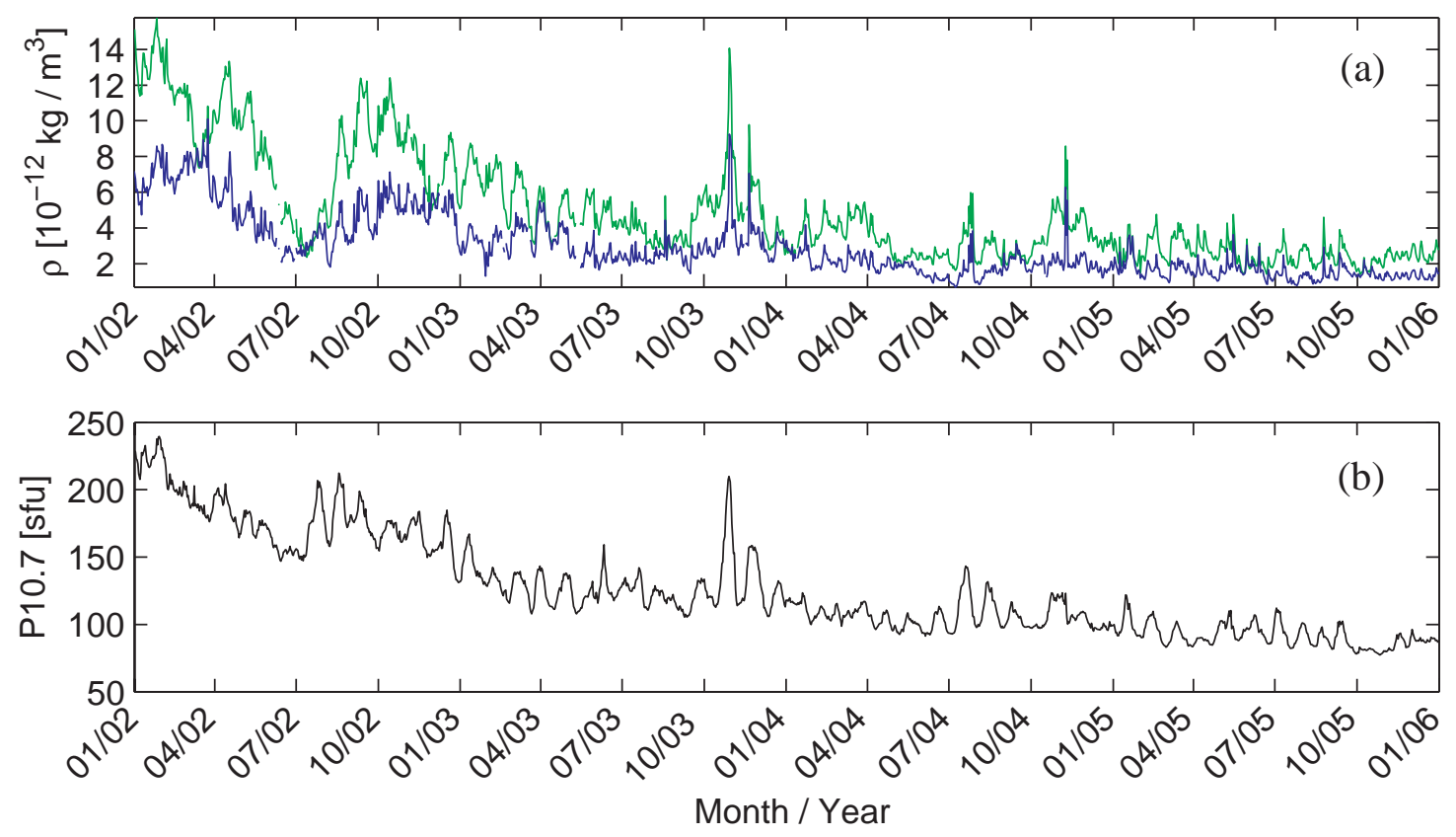

Fig. 2. (a) Daily mean density variations for the considered period (2002-2005). The green curve shows air density on the day side and the blue curve on the night side. (b) Plot of the solar flux variation (P10.7) for the same period.

\subsection{Dependences of density on solar flux}

Our aim is to delineate the various dependences of the thermospheric densities on external influences. For that reason we try to use a suitable data selection emphasising the response to a particular parameter. In order to avoid a seasonal bias the data interval is just four years. Local time dependences are considered by treating day time and night time data separately. Figure 2 shows the observed temporal variation of the longitudinally averaged daily mean air density over the whole four years period. There are separate curves for day and night data. For reference, the evolution of the solar flux index, P10.7, is plotted in the lower part of the figure. It is quite evident that the density tracks closely the changes in solar flux. This is valid for both the long-term variation, as well as for the 27-day solar rotation signal. Densities observed on the day side are about twice as high as on the night side, but both exhibit similar variations.

In order to investigate the dependence on the solar flux level more quantitatively we have performed a correlation analyses separately for the day and night side data set. In this case only data from magnetically quite days with $A_{p}<15$ were considered. This should help to avoid a mixing of the different influences.

Results of the correlation are shown in Fig. 3. For both local time sectors we obtain high degrees of correlation $(r \sim 0.9)$ between the daily averaged low-latitude density and the P10.7 value of the previous day. Motivated by the favourable result we computed the regression lines for both cases. From the equations describing the regression lines (given in the frame) we see that the slope on the day side is larger by a factor of 2 than that on the night side. This means the sensitivity of the air densities on EUV input is dependent on local time but not on the level of the solar flux at least over the considered range, P10.7=80-240.

\subsection{Local time dependence of air density}

As a next step of our statistical analysis we looked into the local time variation of the thermospheric density. In a previous paper Liu et al. (2005) have studied the global distribution of the thermospheric density in some details. As part of their work they show in Fig. 8 the mean diurnal variation of the mass density near the equator. From that we see how the density changes from day to night. In this study we tested the ratio of the low latitude density observed on the upleg versus that from the downleg part of the orbit. Corresponding samples are separated by $12 \mathrm{~h}$ in local time (e.g., day vs. night; morning vs. evening). Figure 4 shows a time series of the ratio over all years. Here again only quiet days $\left(A_{p}<15\right)$ have been considered. The ratio exhibits flat plateaus around a factor of 2 and shows triangular decreases down to one. The ratio of 2 is achieved when comparing day and night time densities and the approach to 1 is expected at the transition between day and night. Interestingly, the minimum ratio $(\sim 1)$ is not achieved when comparing 06:00 LT 
(a) CHAMP, 2002-2005, Ap $<15$

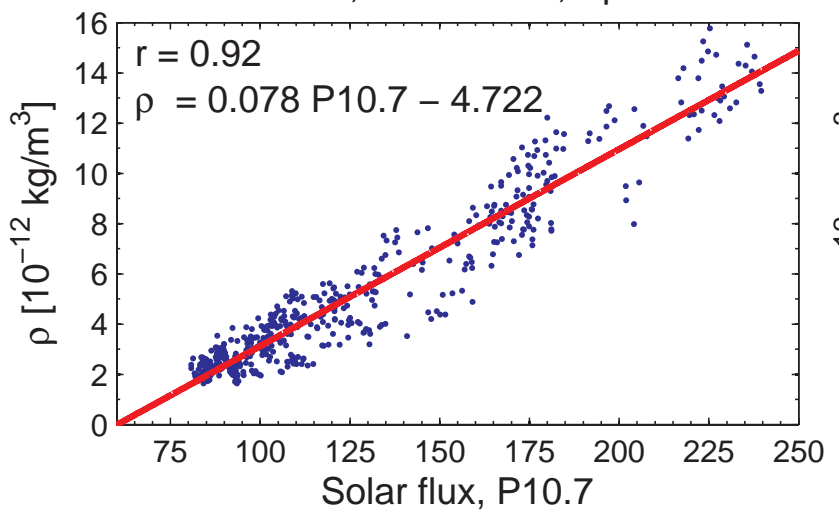

(b) CHAMP, 2002-2005, Ap $<15$

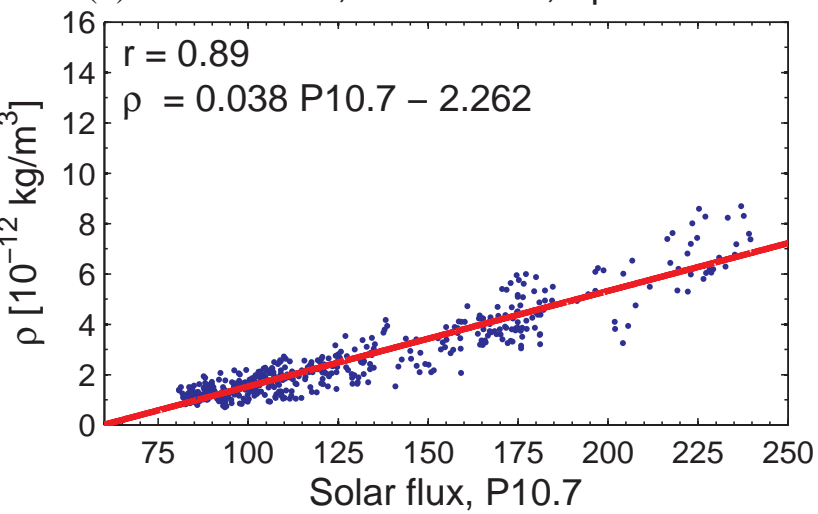

Fig. 3. Correlation between CHAMP-derived densities and the solar flux level (P10.7), (a) for the day side (10:30-16:30 LT) and (b) for the night side (22:30-04:30 LT) density. The density readings are longitudinally averaged daily means.

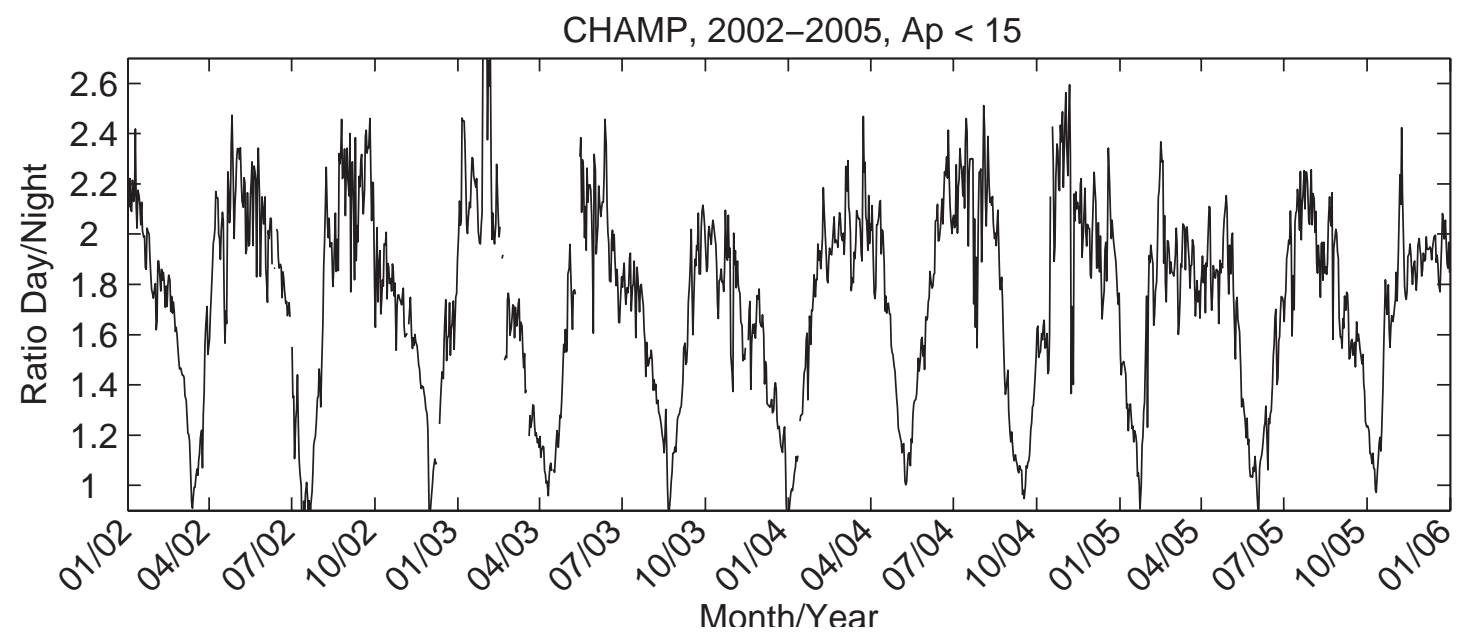

Fig. 4. Time series of the ratio between the day and night side densities for quiet days $\left(A_{p}<15\right)$. The density readings are longitudinally averaged daily means.

with 18:00 LT observations, but it results from the ratio at 07:30 LT vs. 19:30 LT. There seems to be a delayed response of the thermosphere by $1.5 \mathrm{~h}$ to the solar input. Based on the variation of the ratio, as presented in Fig. 4, we have defined in this study the local time interval 10:30 to 16:30 LT as day side data and 22:30 to 04:30 LT as night side data. The hours in between are considered as transition periods. This grouping of local time sectors is used consistently throughout the paper.

From Fig. 4 we got the impression that the density ratio between day and night does not change much with time. This implies that the significant decrease of the solar flux P10.7 by about a factor of 3 during the 4 years considered here has not a great influence on the density ratio. In order to test that suggestion quantitatively we performed a correlation analysis of the ratio between the day and night side densities versus P10.7. As can be deduced from Figure 5, there is no sign of a dependence over the whole range of $\mathrm{P} 10.7=80 \ldots$ 240. We regard this as a significant and important result.

In another analysis we checked this density ratio in dependence on local time and season. Figure 6 shows the local time variation of the day-to-night ratio over the above defined local time sector (10:30-16:30 LT) separately for the four seasons. Here again we have limited our data selection to quiet days $\left(A_{p}<15\right)$. As can be seen from Fig. 6, all four seasons show the same pattern. Although the standard deviation is significant, we obtain rather similar mean ratios for March equinox, June solstice, September equinox, December solstice of $1.92,2.03,1.99,1.93$, respectively. The small differences cannot be regarded as significant, given the observed variance. There is a general trend of lower ratios during the earlier hours increasing by about $6 \%$ towards the end of the interval. 


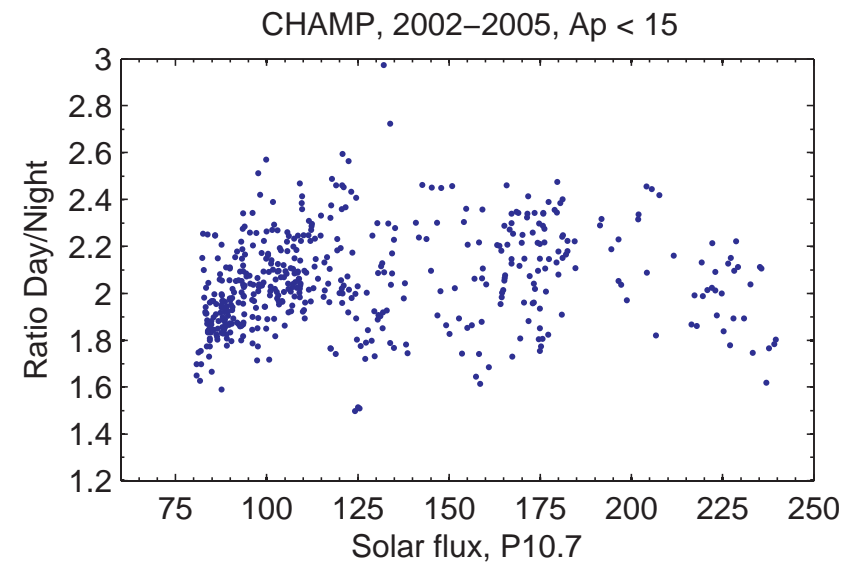

Fig. 5. Ratio between the day and night side densities versus solar flux level. Only days with $A_{p}<15$ have been considered. There is practically not dependence on solar flux observed.

\subsection{Seasonal dependence of air density}

Since we have limited our attention to the low latitudes no big seasonal variation of the solar zenith angle and related effects is expected. For that reason we have paid special attention to avoid contamination by other dependences. First, the data are normalised to a common height. Second, fixed local time intervals are used (10:30-16:30 LT and 22:3004:30 LT). Third, only data from quiet days are selected $\left(A_{p}<15\right)$, and fourth, the dependence on solar flux is removed by normalisation to a flux level of P10.7=130. For this latter procedure we make use of the regression line equations given in Fig. 3. For example, the density normalisation for the day side reads

$\rho(130)=\rho(P 10.7) \frac{0.078 \cdot 130-4.722}{0.078 \cdot \mathrm{P} 10.7-4.722}$

where $\rho(\mathrm{P} 10.7)$ is the recorded density.

Figure 7 shows the obtained density variations in the day and night sectors after normalisation to the constant solar flux level. When comparing to Fig. 2 we see that the long-term trend as well as the 27-day variation disappear. At certain intervals the observations deviate, however, systematically from the mean value.

For identifying a possible seasonal dependence of the density we resorted the readings. All four years were taken together and the data ordered by day of year (DoY). As can be seen in Fig. 8, systematic variations show up now over the course of a year, both in the day and night side observations. In order to get a quantitative description of the variations we fitted harmonic annual, semi-annual and ter-annual functions to the quiet-time data displayed in Fig. 8. Any further expansion to higher harmonics did not improve the fit.

Since the data stream is not continuous a simple FFT cannot be applied. The alternatively used fitting procedure (us-
Table 1. Coefficients of the harmonic seasonal variation functions. Prevailing conditions are $a_{p}<15, \mathrm{P} 10.7=130, h=400 \mathrm{~km}$. The uncertainty within a $95 \%$ confidence is about $\pm 2.4 \times 10^{-14} \mathrm{~kg} / \mathrm{m}^{3}$ and $\pm 1.6 \times 10^{-14} \mathrm{~kg} / \mathrm{m}^{3}$ for the amplitude of all the harmonics on the day and night side, respectively. The uncertainty of the phases (day of peak amplitude) is about \pm 5 days.

\begin{tabular}{lllll}
\hline & \multicolumn{2}{c}{ Day side } & \multicolumn{2}{c}{ Night side } \\
& $\begin{array}{l}\text { Amplitude } \\
{\left[10^{-12} \mathrm{~kg} / \mathrm{m}^{3}\right]}\end{array}$ & $\begin{array}{l}\text { Phase } \\
{[\text { DoY }]}\end{array}$ & $\begin{array}{l}\text { Amplitude } \\
{\left[10^{-12} \mathrm{~kg} / \mathrm{m}^{3}\right]}\end{array}$ & $\begin{array}{l}\text { Phase } \\
{[\text { DoY }]}\end{array}$ \\
\hline annual & 0.77 & 23 & 0.45 & 25 \\
semi-annual & 0.70 & 97 & 0.37 & 96 \\
ter-annual & 0.24 & 52 & 0.11 & 38 \\
constant & 5.57 & - & 2.72 & - \\
RMSE & 0.75 & - & 0.50 & - \\
\hline
\end{tabular}

ing sine and cosine) provides reliable and consistent results. The obtained functions for day and night are overplotted in Fig. 8. Peak densities are encountered at equinox seasons, with highest values reached on DoY 83. Lowest values are found around June solstice (DoY 192). The ratio between the extremes is 1.63 . The thermosphere performs primarily annual and semi-annual variations with comparable amplitudes. The fairly weak ter-annual harmonic causes just a slightly higher maximum in spring compared to that in autumn. Annual and semi-annual curves are in phase around June solstice, thus resulting in a deep minimum. Very similar variations are observed on the day and night side. As expected, the ratio between the fitted functions provides an almost constant value close to 2 over the whole year. This confirms the results of Fig. 6, where we have already found the seasonal independence of the day/night ratio. Table 1 lists the details of the annual harmonic functions, $\left(A_{n} \cos \left(n \omega t-\phi_{n}\right)\right)$, where $A_{n}$ reflects the amplitudes of the density variations and $\phi_{n}$ the phase in DoY; the index $n$ takes the value 1 for annual, 2 for semi-annual and 3 for ter-annual harmonics. Also the RMSE of the residuals between the data and the fitted function is given in Table 1 .

\subsection{Dependence on magnetic activity}

So far we have limited our analysis to magnetically quiet days. Now we are going to focus on the dependences of the equatorial thermospheric density on the magnetospheric input. We use the indices $a_{p}$ and $a_{m}$ for parameterising the magnetic activity. In order to remove other effects we use data normalised to $400 \mathrm{~km}$ altitude, to $P 10.7=130$, and we subtract the seasonal signal as quantified in Table 1. For quiet days we thus get a data set that is varying around zero. Subsequently, these data are termed density residuals.

Menvielle et al. (2007) propose another approach for removing the non-magnetospheric influences. The solar flux effect is accounted for by normalising the density data to a fixed level with the help of the NRLMSISE-00 model. 

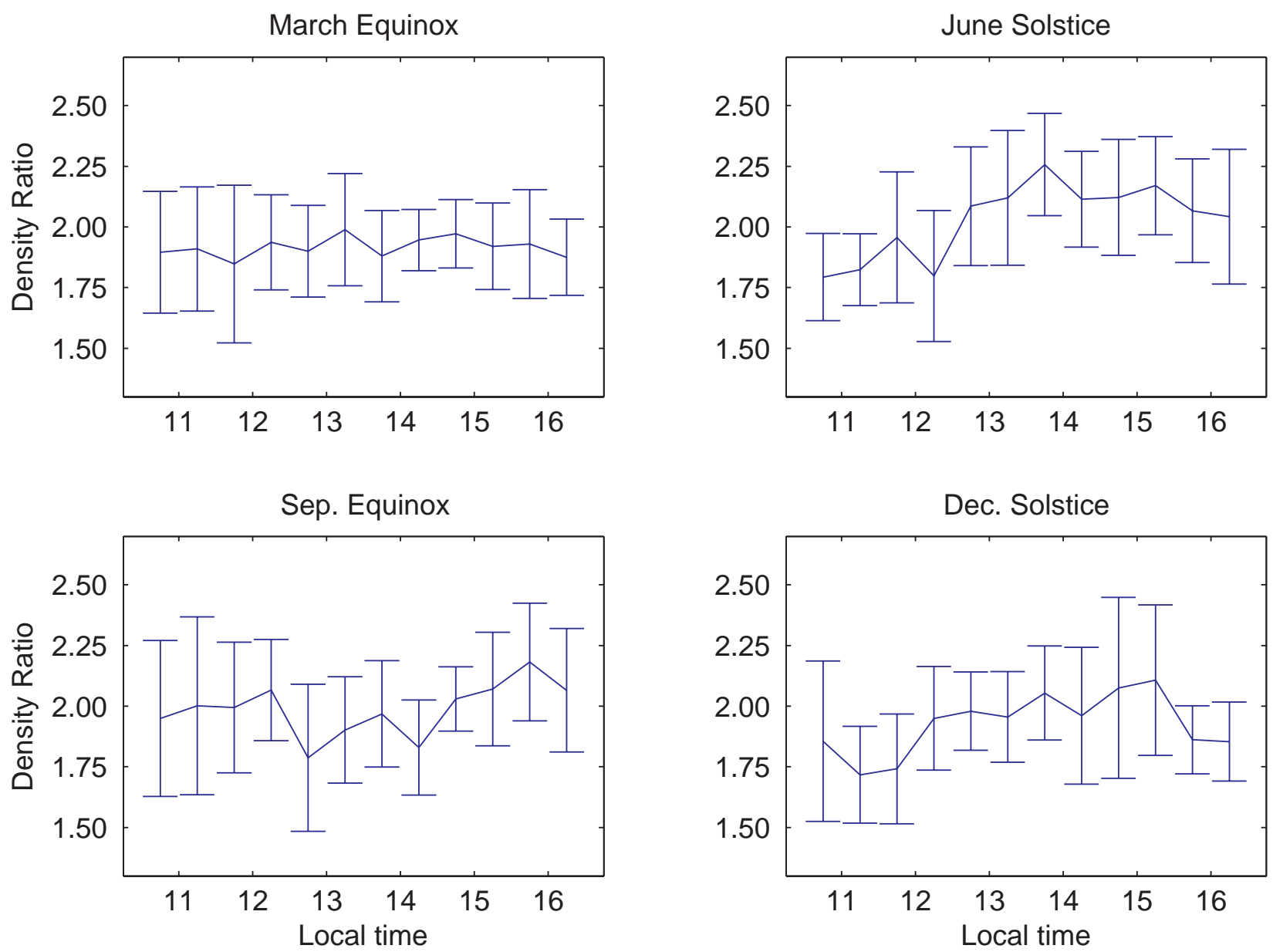

Fig. 6. Local time variation of the day-to-night density ratio separately for the four seasons. Only quiet days with $A_{p}<15$ have been considered. The error bars represent the standard deviation of the half-hours bins.

Instead of using F10.7 they prefer a replacement based on the MgII index. For removing the density variations with altitude they apply a singular value decomposition approach. This provides them with a typical background variation pattern along the orbit. For the four-year analysis presented here we prefer our approach. Since the encountered density variations due to solar flux changes are much larger than those caused by altitude variations, we have removed only the latter with the help of a model.

It is well known that magnetic activity causes an enhancement of the thermospheric density. The effect occurs, however, not simultaneously on the day and night side. For determining a possible delay time we performed a crosscorrelation between the densities in the two time sectors. In this case we are interested in the response to magnetic activity, thus select data from 3-h intervals with $a_{p}>15$. The correlation coefficient, $r$, depending on the delay, $d$, is calculated as: $r(d)=\frac{\sum_{i}\left[\left(x_{i}-\bar{x}\right) \times\left(y_{i-d}-\bar{y}\right)\right]}{\sqrt{\sum_{i}\left(x_{i}-\bar{x}\right)^{2}} \sqrt{\sum_{i}\left(y_{i-d}-\bar{y}\right)^{2}}}$

where $x_{i}$ and $y_{i}$ are the density values on the day and night sides, respectively, and $\bar{x}, \bar{y}$ are the mean values. The delay time, $d$, is given in multiples of the orbital period ( $\sim 93 \mathrm{~min}$ ). Results of the correlation analysis are shown in Fig. 9. As can be seen from the graph, the day and night side densities are highly correlated. The coefficient peaks at a delay between 0 and 1 orbital period with $r=0.79$. This means, the night side thermosphere reacts later. For estimating the average delay time we have to take into account that the day side is always sampled before the night side; contributing half of an orbital period from the sampling. All together, the density bulge at the equator caused by magnetospheric input shows up 1 to $2 \mathrm{~h}$ later on the night side than on the day side. This time shift has to be taken into account when investigating the response to magnetospheric driving.

In a next step we want to find out, how the equatorial density is related to the magnetic activity indices. Here it 


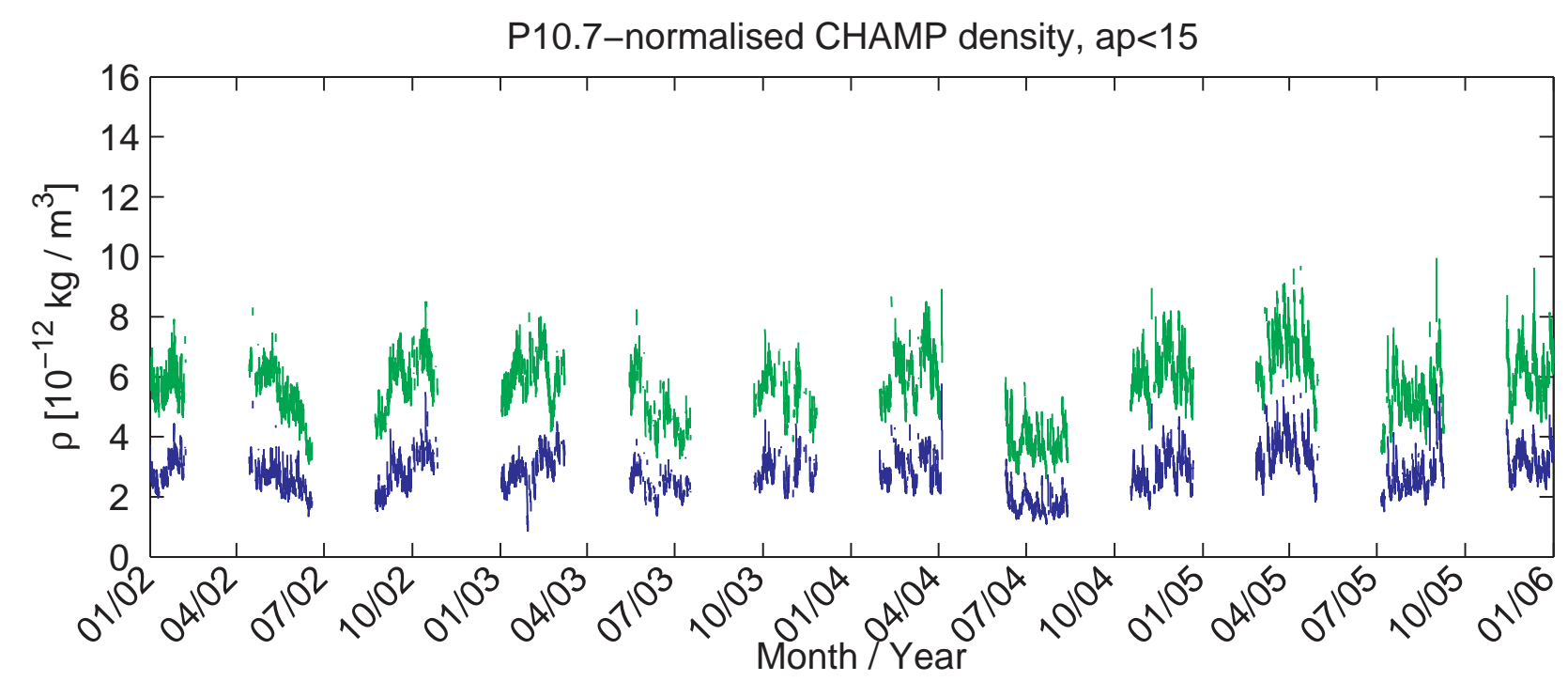

Fig. 7. Density variations in the day (green) and night (blue) time sector after normalisation to a constant solar flux level of P10.7=130. Only quiet 3-h intervals have been considered $\left(a_{p}<15\right)$.
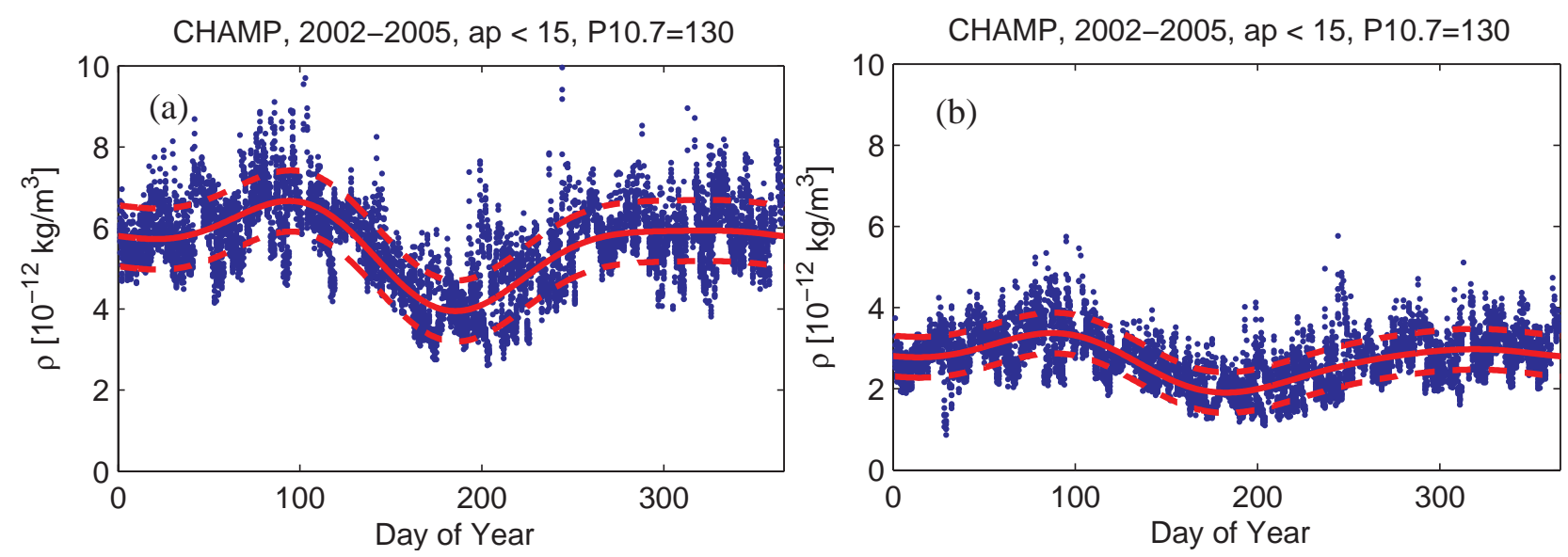

Fig. 8. Seasonal variation of the density in the day (a) and night (b) time sector. The same data as for Fig. 7 have been used. The solid red curves represent the fitted annual harmonics functions. Dashed lines mark the RMSE range.

is again important to identify a possible delayed reaction of the thermosphere to changes of the indices. In order to get a synchronous data set of indices and density values the 3$\mathrm{h}$ indices have been resampled by linear interpolation to the times of the CHAMP readings. The cross-correlation is again performed according to Eq. (5). In this case the $x_{i}$ represent the indices and $y_{i}$ the density values.

Figure 10 shows the result of the cross-correlation between density enhancement and the activity index $a_{p}$. We are focussing here on the night side results since the peak correlation coefficient of the night side $(r=0.64)$ is significant higher than that on the day side $(r=0.55)$. From the graph in Fig. 10 we can read a delay of 3 orbital periods $(4.5 \mathrm{~h})$.
For the subsequent correlation of the observed densities with the indices we consider a delayed response on the day side by $3 \mathrm{~h}$ and on the night side by 4 to $5 \mathrm{~h}$.

As a next step we tried to identify the sensitivity of the equatorial density to magnetic activity. Properly delayed readings were correlated (separately) with the magnetic activity indices $a_{p}$ and $a_{m}$. First we sorted the density residuals in 6 logarithmically spaced $a_{p}, a_{m}$ bins, separately for day and night side readings. The median value of the density residuals from each bin is plotted in Fig. 11.

As can be seen, the graphs suggest a linear relation between air density enhancement and magnetic activity. The obtained slopes are similar for $a_{p}$ and $a_{m}$. In case of the $a_{p}$ 


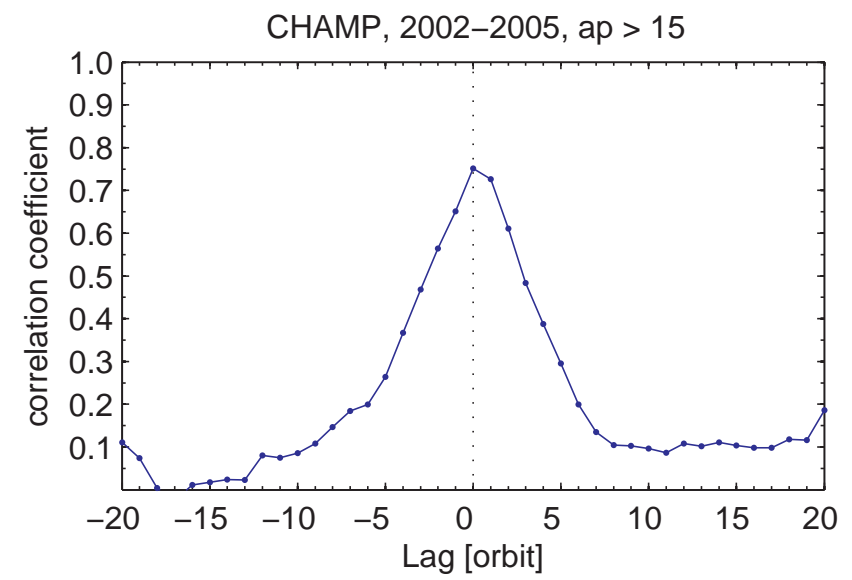

Fig. 9. Cross-correlation between the density readings on the day and night side for magnetically active 3 -h intervals $\left(a_{p}>15\right)$. Positive lags indicate a delayed response on the night side.

index we find slightly different slopes on the day and night sides. Obviously, on the night side the sensitivity is higher by $20 \%$. Conversely, in case of the $a_{m}$ index the slopes for day and night are the same. Furthermore, both $a_{m}$ curves go through the origin. The correlation analysis provides slightly higher coefficients, $r\left(a_{p}\right)=0.995$ and $r\left(a_{m}\right)=0.997$, and the variance of the mass density values within a bin is also smaller in case of the $a_{m}$ sorting. From these results we may conclude that $a_{m}$ is preferable over $a_{p}$ for parameterising the magnetospheric input to the thermosphere.

\section{Discussion}

In this study we have performed statistical analyses on thermospheric mass density data in order to identify its response to the various drivers. The CHAMP observations considered are from the declining phase of the solar cycle 23 (20022005). These accelerometer measurements have earlier been interpreted by several other groups. We thus will discuss our results in comparison with previous findings.

One important issue of our study is the separation of the different effects in order to identify the dependences on the various parameters. Our approach is to make as little as possible use of existing atmospheric models but find other ways of isolating effects.

It is well-known that the atmospheric density depends strongly on altitude. Since the CHAMP orbit is slowly decaying over the years, we have normalised our data to a reference height of $400 \mathrm{~km}$. This is the only case where the NRLMSISE-00 model is applied. We regard the altitude dependence at low latitudes as a well modelled quantity, at least over an altitude range of less than one scale height. Liu et al. (2007) had quoted the uncertainty introduced by this kind of normalisation to be of the order of $5 \%$.

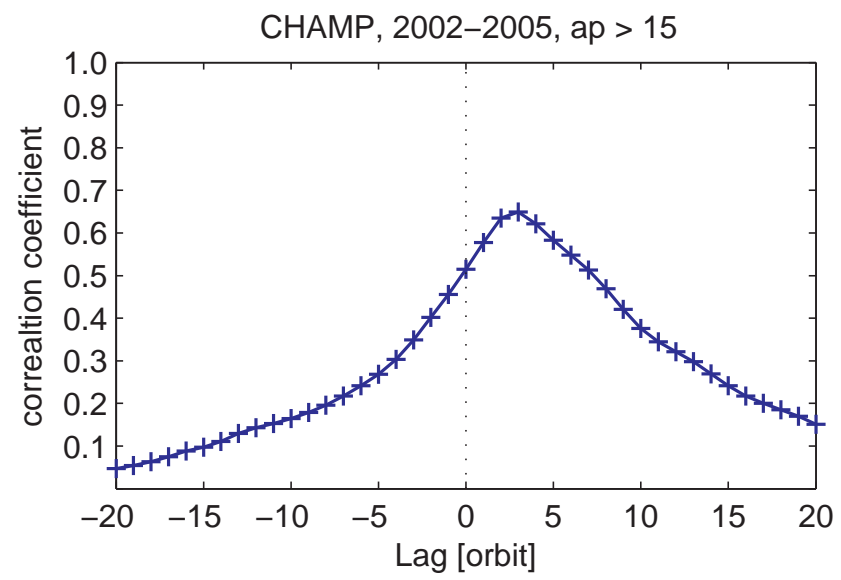

Fig. 10. Cross-correlation between the activity index $a_{p}$ and the density variations on the night side. Positive lags indicate a delayed response of the density.

\subsection{Dependence on solar flux level}

It is quite obvious that the thermospheric density depends strongly on the flux of solar EUV radiation. From Fig. 2 we can read that this is valid both for the day and night side. The burning question is which is the best proxy to describe the relevant solar flux appropriately? Guo et al. (2007) have performed an extensive study on the relation between solar indices and density (see also their many references on that topic). They tested many combinations of proxies. We adopted their Trial 1 (Table 3) which does not give the best but reasonable results. It is practically identical with our P10.7, the equally weighted sum of the daily F10.7 value and its 81-day mean. Liu et al. (2006) confirmed that P10.7 is also a suitable proxy for the solar flux in ionospheric studies.

As can be read from Fig. 3 our applied P10.7 parameter is highly correlated with the thermospheric density during magnetically quiet days. It is interesting to note that the density increases over proportionally to P10.7 for solar flux levels above $80 \mathrm{sfu}$.

Another result from Fig. 3 is that the slope of the regression line is twice as high during day time as during the night. This means, the sensitivity to solar forcing at low latitude is local time dependent. Guo et al. (2007) also investigated dependences of the CHAMP-derived thermospheric density on solar flux level. They, unfortunately, did not differentiate their results by local time. Since the CHAMP orbit is precessing slowly through local time ( $1 \mathrm{~h}$ per 11 days), a beating will occur between the flux variations due to solar rotation and local time dependent sensitivity of the thermosphere. This modulation of the solar input is probably a reason for the apparent harmonics of solar rotation in the spectrum of density readings (their Fig. 4). 
CHAMP, 2002-2005, P10.7=130, Delay: 3 or 4.5 h

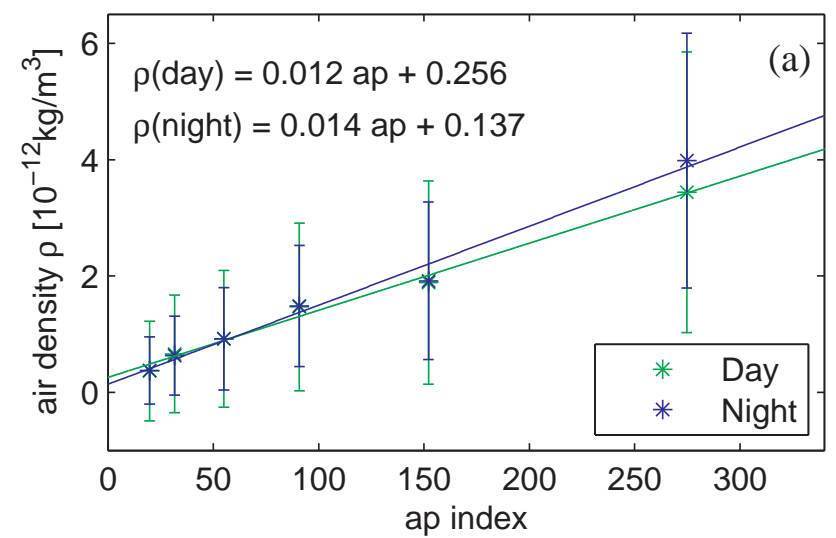

CHAMP, 2002-2005, P10.7=130, Delay: 3 or $4.5 \mathrm{~h}$

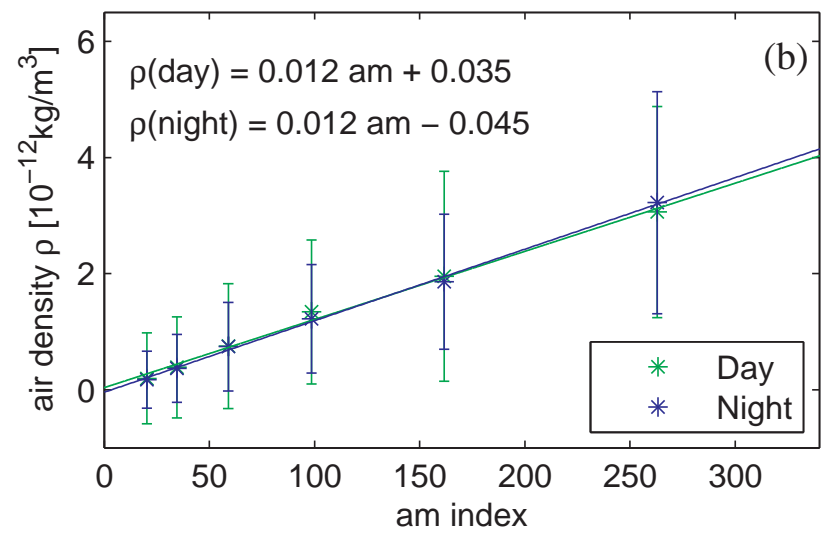

Fig. 11. The dependence of the density on magnetic activity, as represented by the indices $a_{p}$ and $a_{m}$, is shown in frames (a) and (b), respectively. A linear fit to the medians of each bin was applied (solid lines). The error bars indicate the standard deviation of the values within a bin.

\subsection{Local time dependence}

As is obvious from Figs. 2 and 3, the thermospheric density strongly depends on local time. Interestingly, we find for the ratio day (10:30-16:30 LT) to night (22:30-04:30 LT) a factor very close to 2 during magnetically quiet days. This ratio is independent of the solar flux level and shows no seasonal variation. We regard it as an important constraint for the design of thermospheric models.

This specific factor of 2 characterises the difference in sensitivity between day and night sides to solar input. Interestingly, this is not only valid for the EUV flux over the whole range considered (P10.7=80-240) but also for the annual and semi-annual variations. During the hours around dawn (04:30-10:30 LT) and dusk (16:30-22:30 LT) the sensitivity to solar flux varies almost linearly between the day and night side values. This local time characteristic of the thermosphere has to be taken into account when investigating any of the dependencies on solar input.

\subsection{Seasonal dependence}

For identifying a seasonal dependence of the thermospheric density it is necessary at first to remove the influence of the solar flux variations. We applied in Eq. (4) the dependence as determined from the correlation analysis in Fig. 3 rather than using an atmospheric model for normalising to the constant flux level of P10.7=130. It is obvious from Fig. 8 that the low latitude thermospheric density performs an annual and semi-annual variation. There is no justification found in our quiet-day data for considering higher annual harmonic terms.

We think, the study by Bowman (2004) cannot directly be compared with ours since the author did not remove the local time, solar flux and magnetic activity effects on the thermospheric mass density before analysing the seasonal variation.
The purpose of that study is to show the overall semi-annual variation as is for all the years considered. After removing all the other influences we do not find any significant yearto-year changes of the seasonal variation within the 4 years considered. The ratio we obtain between minimum and maximum of 1.6 for a medium flux level of 130 sfu corresponds to the $60 \%$ variation reported by Bowman (2004) for solar minimum conditions. The results presented here are regarded to be more general, and they should be useful for modellers who need to parameterise all the dependences separately.

Different from the paper above Guo et al. (2008) employ mass density data also derived from CHAMP observations for the same 4 years as this study. They account for all the various influences on the density by subtracting model predictions from each sample. Unfortunately, they found an incomplete removal of the local time and solar flux effects. The persistent 27-day signal in the residuals, for example, is an indication for a remaining solar flux influence on the analysed data. The authors have removed the 27-day signal by running averages over 27 days, but this does not prevent the density residuals from being contaminated by the long-term EUV variations. It is therefore difficult to judge which part of the intra-annual spectral variations is caused by the imperfection of the applied model and which by the influence of external forcing. Different from Guo et al. (2008) we did not rely on model predictions for isolating the different forcing terms. Our results show the clear dominance of the annual and semi-annual harmonics, and they provide no indication for a year-to-year change when constant solar flux levels are considered.

Largest densities are encountered during the two equinoxes, lowest occur during June solstice. In particular the March equinox exhibits peak densities. During June solstice both the minima of the annual and semi-annual 
variations add up causing the absolute minimum. Since the two harmonics are out of phase in December we find only a gentle variation there. This difference between the solstices leads to very different thermospheric seasonal variations in the polar regions of the two hemispheres. The June minimum coincides with the polar winter in the south, causing an additional reduction of the density there, while during December solstice the continuous sunshine further enhances the globally higher density. Thus we can expect a large annual variation in the southern polar region. Conversely, in the northern polar region the solar forcing is out of phase with the global variation. This results in a much smaller annual oscillation. An indication of these hemispheric differences has been presented by Liu et al. (2007) in their Fig. 4.

The annual variation of the thermospheric density is a well known global phenomenon first deduced from satellite orbit analysis (e.g., King-Hele and Walker, 1969). Its maximum occurs one month after December solstice. Partly it may be due to the eccentricity of the Earth's orbit around the sun with the perihelion in early January. But so far there is no conclusive explanation for its full amplitude. Interestingly, a similar annual variation is observed in total electron content of the ionosphere (Mendillo et al., 2005; Zhao et al., 2005; Rishbeth and Müller-Wodarg, 2006). Probably, both phenomena are related.

The semi-annual variation attains its maxima shortly after the equinox dates. We do not relate these maxima in density to the enhanced magnetic activity during the equinox seasons (Russell and McPherron, 1973). The data considered here are all from quiet days. Furthermore, magnetosphere-driven density enhancements exhibit a day-to-night ratio different from 2. An alternative explanation is based on a change of thermospheric composition. The large-scale wind systems differ between solstice and equinox. During equinox they provide a stronger mixing of the air. Fuller-Rowell (1998) proposed the so-called "spoon mechanism" as an explanation for the enhanced density during equinox seasons.

The semi-annual variation in air density has earlier been deduced from air drag variations of orbital objects (e.g., Moore, 1983). Also Liu et al. (2007) confirmed this kind of seasonal variation in their study on the equatorial mass density anomaly. We favour for the high densities during equinoxes the explanation offered by Fuller-Rowell (1998) which is based on a higher $\mathrm{N}_{2} / \mathrm{O}$ ratio. But this mechanism cannot account for the very low density at June solstice.

\subsection{Dependence on magnetic activity}

So far we have investigated the solar forcing of the thermosphere. For that we had considered only data from quiet days $\left(A_{p}<15\right)$. Now our interest is focussed on the data from active days. For a clear isolation of the magnetospheric input all the solar driven influences had been removed first. This means, the density residuals considered vary around zero for quiet days. Any observed enhancement due to magnetic ac- tivity has to be regarded as an additive contribution to the background density.

An important consideration in this context is the delay time after which the thermosphere at $400 \mathrm{~km}$ responds. At first we found that the time interval is local time dependent. The density enhancements occur 1 to $2 \mathrm{~h}$ earlier on the dayside than on the night side. This observation is consistent with the delayed response of the equatorial thermosphere on the night side as reported by Liu and Lühr (2005) for the big magnetic storms in autumn 2003. Further evidence for that is provided by Wang et al. (2006) who found for the same storms that the field-aligned current (FAC) intensity on the dayside followed quite closely the variation of the solar wind input (when allowing for a $15 \mathrm{~min}$ propagation time from the magnetopause to the ionosphere). Conversely, the FACs on the night side track better the change of the $D_{s t}$ index. The latter reaches its negative peak about $2 \mathrm{~h}$ after the solar wind input. It has to be noted that the delay time is not only a function of local time but depends also on latitude. Since the energy input occurs at auroral latitudes (e.g., Lühr et al., 2004; Rentz and Lühr, 2008), the thermospheric response time is shorter at higher latitudes, and the atmospheric disturbance travels then equatorward. Taking the local time and latitude effects together it can be concluded that the equatorial thermosphere on the night side exhibits the longest delay times.

In a dedicated analysis we have determined the response time of the thermosphere to changes of the magnetic activity index, $a_{p}$. On the night side the cross-correlation peaks at a lag of 3 orbital periods ( $4.5 \mathrm{~h}$ ) (cf. Fig. 10). By considering also the delay between day and night (cf. Fig. 9) we find that the equatorial thermosphere responds to changes in $a_{p}$ with a delay of $3 \mathrm{~h}$ on the day side and 4 to $5 \mathrm{~h}$ on the night side.

When correlating the enhancement of the air density at $400 \mathrm{~km}$ altitude point-by-point with the magnetic activity indices we find a large scatter of the values. Obviously, the $a_{p}$ and $a_{m}$ indices are not too well suited to predict the details of the thermospheric response. In a climatological sense these indices can, however, be used to parameterise the magnetospheric input. For that reason we have first sorted the density readings into $a_{p}, a_{m}$ bins and then considered only the bin medians in the correlation. For both indices we get clear linear response functions. Interestingly, the $a_{m}$ index provides higher correlation coefficients and gives identical slopes for the density enhancements on the day and night side. Also the crossing of both curves through the origin indicates that $a_{m}$ is more suitable for parameterising the magnetospheric input to the thermosphere. Obviously, the observatories used for deriving the $a_{m}$ index are better distributed for estimating the global magnetic activity level than the $a_{p}$ stations.

Lathuillère et al. (2008) earlier investigated the response of the thermospheric density to magnetic activity. They also identified $a_{m}$ as the better index. Based on CHAMP accelerometer data of the year 2004 they determined among others the relative enhancement of the air density on the day and night side in response to magnetic activity. The relative 
increase was found to be higher during night by a factor of 1.66 for $a_{m}=150$. We find that the additive enhancement is the same on the day and night side. Since the relative enhancement depends on the prevailing background density, we cannot compare the results of the two studies directly. But because the density on the day side is always higher than on the night side, an equal additive enhancement due to magnetic activity will result in a larger relative increase in air density on the night side. Thus, their results are qualitatively confirmed by our analysis.

\section{Summary and conclusion}

Based on 4 years (2002-2005) of data sampled by the CHAMP accelerometer we have investigated the characteristics of the thermosphere at low and equatorial latitudes. This region was selected since it is least affected by the seasonal and solar zenith angle variations. Here the global features of the upper atmosphere show up most clearly.

Our aim was to identify and delineate all the main drivers of the thermospheric density. The approach we used is to isolate the response to a certain parameter by data selection and/or removal of the influence from other parameters. We succeeded in deriving quantitative relations for the dependences on all the parameters. For observations at $400 \mathrm{~km}$ altitude we obtained:

1. The dependence on solar EUV flux can be described within the range $\mathrm{P} 10.7=80 \ldots 240$ by a linear function.

day side: $\rho=0.078$ P10.7 -4.72

night side: $\rho=0.038$ P10.7-2.26

Here and below $\rho$ is given in $10^{-12} \mathrm{~kg} / \mathrm{m}^{3}$.

2. A clear annual and semi-annual variation of the density has been identified. An additional ter-annual harmonic makes only a marginal contribution. Details of the coefficients are listed in Table 1. The annual and semi-annual variations have similar amplitudes. Both of them amount to approximately $15 \%$ of the average background density. Density peaks are reached during equinoxes. The lowest densities occur around June solstice. The deduced ratio of the seasonal maximum to minimum is 1.6 .

3. The ratio between the density on the dayside (10:30$16: 30 \mathrm{LT})$ and night side (22:30-04:30 LT) is $2 \pm 0.1$ for all kinds of solar forcings. It is thus independent of solar flux level and seasonal variation. The magnetospheric input changes this constant ratio.

4. For characterising the magnetospheric input the $a_{m}$ activity index has been identified to be a suitable parameter, superior to $a_{p}$. A delayed response of the equatorial thermosphere to variations of $a_{m}$ by $3 \mathrm{~h}$ on the day side and 4 to $5 \mathrm{~h}$ on the night side is observed. When normalised to a fixed solar flux level $(\mathrm{P} 10.7=130)$ and when the seasonal variation is removed, an additive linear increase of the air density with $a_{m}$ is observed. The functional dependence for both the day and night sides is

$$
\Delta \rho=0.012 a_{m}
$$

This means, the absolute density increase due to magnetic activity is the same in both time sectors. But since the quiet-time density on the night side is lower than on the day side, the relative density enhancement in response to magnetospheric input is larger on the night than on the day side.

From all these relations we may conclude that the four considered forcing terms can be treated as linear combinations for parameterising the thermospheric density response.

After having verified the approach of delineating the various thermospheric forcing terms at low latitudes this method can also be applied to other latitudes and local times for a full characterisation of the thermospheric variability.

Acknowledgements. The operational support of the CHAMP mission by the German Aerospace Centre (DLR) and the financial support for the data processing by the Federal Ministry of Education and Research (BMBF), as part of the Geotechnology Programme, are gratefully acknowledged. One of the authors (S.R.) was supported by the Deutsche Forschungsgemeinschaft, DFG, through the Priority Programme "CAWSES", SPP1176.

Topical Editor C. Jacobi thanks J. Guo and another anonymous referee for their help in evaluating this paper.

\section{References}

Bowman, B. R.: The Semiannual Thermospheric Density Variation From 1970 to 2002 Between 200-1100 km, AGU Fall Meeting Abstracts, p. A4, 2004.

Bowman, B. R., Tobiska, W. K., Marcos, F. A., and Valladares, C.: The JB2006 emperical thermospheric density model, J. Atmos. Solar-Terr. Phys., 70, 774-793, 2008.

Bruinsma, S., Tamagnan, D., and Biancale, R.: Atmospheric densities derived from CHAMP/STAR accelerometer observations, Planet. Space Sci., 52, 297-312, doi:10.1016/j.pss.2003.11.004, 2004.

Fuller-Rowell, T. J.: The "thermospheric spoon": A mechanism for the semiannual density variation, J. Geophys. Res., 103, 39513956, doi:10.1029/97JA03335, 1998.

Guo, J., Wan, W., Forbes, J. M., Sutton, E., Nerem, R. S., Woods, T. N., Bruinsma, S., and Liu, L.: Effects of solar variability on thermosphere density from CHAMP accelerometer data, J. Geophys. Res., 112, A10308, doi:10.1029/2007JA012409, 2007.

Guo, J., Wan, W., Forbes, J. M., Sutton, E., Nerem, R. S., and Bruinsma, S.: Interannual and latitudinal variability of the thermosphere density annual harmonics, J. Geophys. Res. (Space Physics), 113, 8301, doi:10.1029/2008JA013056, 2008.

Hedin, A. E.: A revised thermospheric model based on mass spectrometer and incoherent scatter data - MSIS-83, J. Geophys. Res., 88, 10170-10188, 1983. 
King-Hele, D. G. and Walker, D. M. C.: Air density at a height of $470 \mathrm{~km}$ between January 1967 and May 1968 from the orbit of the satellite 1966-118A, Planet. Space Sci., 17, p. 197, 1969.

Lathuillère, C., Menvielle, M., Marchaudon, A., and Bruinsma, S.: A statistical study of the observed and modeled global thermosphere response to magnetic activity at middle and low latitudes, J. Geophys. Res. (Space Physics), 113, 7311, doi: 10.1029/2007JA012991, 2008.

Liu, H. and Lühr, H.: Strong disturbance of the upper thermospheric density due to magnetic storms: CHAMP observations, J. Geophys. Res. (Space Physics), 110, 9, doi:10.1029/2004JA010908, 2005.

Liu, H., Lühr, H., Henize, V., and Köhler, W.: Global distribution of the thermospheric total mass density derived from CHAMP, J. Geophys. Res. (Space Physics), 110, A04301, doi:10.1029/ 2004JA010741, 2005.

Liu, H., Lühr, H., and Watanabe, S.: Climatology of the equatorial thermospheric mass density anomaly, J. Geophys. Res. (Space Physics), 112, A05305, doi:10.1029/2006JA012199, 2007.

Liu, L., Wan, W., Ning, B., Pirog, O. M., and Kurkin, V. I.: Solar activity variations of the ionospheric peak electron density, J. Geophys. Res. (Space Physics), 111, 8304, doi:10.1029/ 2006JA011598, 2006.

Lühr, H., Rother, M., Köhler, W., Ritter, P., and Grunwaldt, L.: Thermospheric up-welling in the cusp region: Evidence from CHAMP observations, Geophys. Res. Lett., 31, L06805, doi: 10.1029/2003GL019314, 2004.

Mendillo, M., Huang, C.-L., Pi, X., Rishbeth, H., and Meier, R.: The global ionospheric asymmetry in total electron content, J. Atmos. Solar-Terr. Phys., 67, 1377-1387, doi:10.1016/j.jastp. 2005.06.021, 2005.

Menvielle, M., Lathuillère, C., Bruinsma, S., and Viereck, R.: A new method for studying the thermospheric density variability derived from CHAMP/STAR accelerometer data for magnetically active conditions, Ann. Geophys., 25, 1949-1958, 2007, http://www.ann-geophys.net/25/1949/2007/.
Moore, P.: The semi-annual variation in air density for 1974-1978 from the orbit of 1972-05B, Planet. Space Sci., 31, 1501-1515, doi:10.1016/0032-0633(83)90022-3, 1983.

Picone, J. M., Hedin, A. E., Drob, D. P., and Aikin, A. C.: NRLMSISE-00 empirical model of the atmosphere: Statistical comparisons and scientific issues, J. Geophys. Res., 107, 1468, doi:10.1029/2002JA009430, 2002.

Prölss, G. W.: Magnetic Storm Associated Perturbations of the Upper Atmosphere, p. 227, Magnetic Storms, Geophysical Monograph Series, vol. 98, 1997.

Reigber, C., Lühr, H., and Schwintzner, P.: CHAMP mission status, Adv. Space Res., 30, 129-134, 2002.

Rentz, S. and Lühr, H.: Climatology of the cusp-related thermospheric mass density anomaly, as derived from CHAMP observations, Ann. Geophys., 26, 2807-2823, 2008, http://www.ann-geophys.net/26/2807/2008/.

Rishbeth, H. and Müller-Wodarg, I. C. F.: Why is there more ionosphere in January than in July? The annual asymmetry in the F2-layer, Ann. Geophys., 24, 3293-3311, 2006, http://www.ann-geophys.net/24/3293/2006/.

Russell, C. T. and McPherron, R. L.: Semiannual variation of geomagnetic activity., J. Geophys. Res., 78, 92-108, 1973.

Wang, H., Lühr, H., Ma, S. Y., Weygand, J., Skoug, R. M., and Yin, F.: Field-aligned currents observed by CHAMP during the intense 2003 geomagnetic storm events, Ann. Geophys., 24, 311324, 2006, http://www.ann-geophys.net/24/311/2006/.

Zhao, B., Wan, W., Liu, L., Yue, X., and Venkatraman, S.: Statistical characteristics of the total ion density in the topside ionosphere during the period 1996-2004 using empirical orthogonal function (EOF) analysis, Ann. Geophys., 23, 3615-3631, 2005, http://www.ann-geophys.net/23/3615/2005/. 